\title{
On Treatment
}

National Cancer Institute

\section{Source}

National Cancer Institute. On Treatment. NCI Thesaurus. Code C142170.

The time during which a patient is receiving treatment. 